\title{
Successful Treatment of a Resistant Subclone in ALK-Rearranged NSCLC
}

\author{
David König $^{a} \quad$ Urs R. Meier $^{c}$ Bernd Klaeser ${ }^{d}$ Spasenija Savic ${ }^{b}$ \\ Miklos Pless ${ }^{\mathrm{e}}$ \\ aDepartment of Medical Oncology, University Hospital Basel, Basel, Switzerland; 'b Institute \\ of Pathology, University Hospital Basel, Basel, Switzerland; 'Department of Radio-Oncology, \\ Cantonal Hospital Winterthur, Winterthur, Switzerland; ${ }^{\mathrm{d} D e p a r t m e n t}$ of Nuclear Medicine, \\ Cantonal Hospital Winterthur, Winterthur, Switzerland; eDepartment of Medical Oncology, \\ Cantonal Hospital Winterthur, Winterthur, Switzerland
}

\section{Keywords}

ALK+ non-small cell lung cancer · Ceritinib - Oligoprogressive disease · Radiotherapy · Resistant subclone

\begin{abstract}
Anaplastic lymphoma kinase (ALK) tyrosine kinase inhibitors (TKIs) are established effective therapies in patients with ALK-rearranged advanced non-small-cell lung cancer (NSCLC). Upon progressive disease, patients normally receive a subsequent ALK TKI. However, when disease progression occurs in a limited number of sites, an oligoprogressive approach is a treatment option. In our case, FDG-PET/CT scan detected a progressive site in a patient with ceritinib therapy. Biopsy of the lesion was not possible because of its location. Progression was therefore confirmed by liquid biopsy with identification of the resistant subclone ALK G1202R. Definitive radiotherapy of the progressive site led to the disappearance of the ALKresistant mutation. Meanwhile, ceritinib therapy was continued. The absence of disease both on repeated imaging and liquid biopsy indicates that eradication of a resistant subclone with an oligoprogressive treatment approach might be possible.

\section{Introduction}

Treatment of patients with anaplastic lymphoma kinase (ALK)-rearranged NSCLC progressing on ceritinib therapy after having received crizotinib depends on symptoms, extend and site of progression, and treatment availability. Retrospective data indicate that 


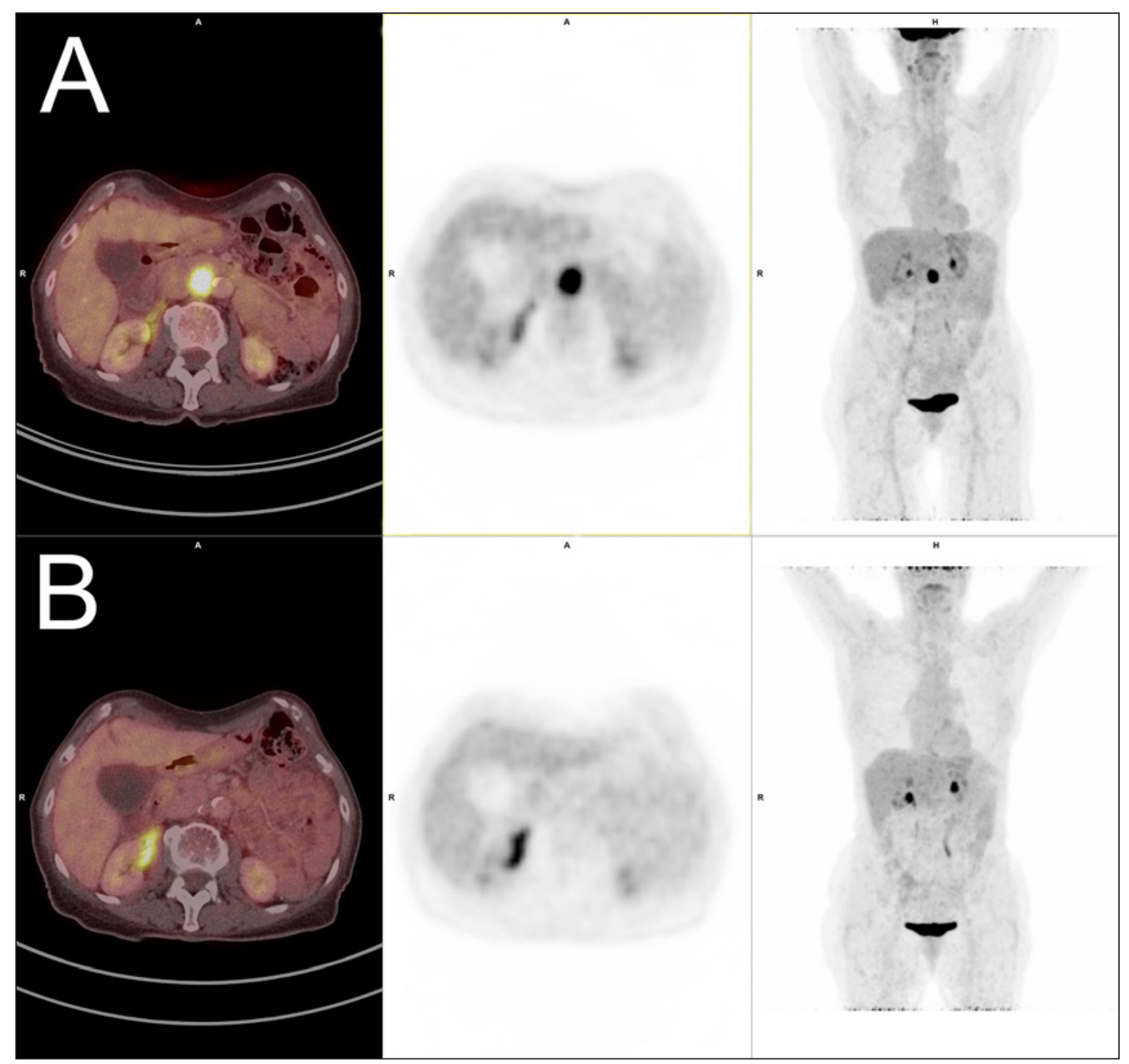

Fig. 1. FDG-PET/CT of the inter-aortocaval lymph node before (A) and after radiotherapy (B).

in selected cases with oligoprogressive disease, tyrosine kinase inhibitor (TKI) treatment continuation with definitive local therapy of the progressing site can be an option $[1,2]$. The rationale of such an approach is maintaining disease control with a well-tolerated treatment, avoiding tumor-seeding from the progressive site, or even eliminating the resistant subclone.

\section{Case Report}

A 66-year-old woman with a diagnosis of inoperable lung adenocarcinoma in 2008 was first treated with platinum-based chemotherapy (partial response). Upon progression, she then received an experimental treatment with an anti-EGFR agent (progressive disease). In 2010, the biopsy of a progressive supraclavicular lymph node (LN) revealed an EMLA4-ALK rearrangement. First-line TKI crizotinib was started and showed a durable response for almost 5 years (partial response). In 2015, the patient presented with progression confined 
to the left axillary and inter-aortocaval LNs. Second-line TKI therapy with ceritinib was initiated with consistent complete response for over 15 months. In 2017, FDG-PET/CT detected disease relapse in a solitary, highly FDG-avid inter-aortocaval LN. Biopsy of the lesion was not possible because of its location. Next-generation sequencing of cell-free circulating tumor DNA (ctDNA) identified an ALK G1202R mutation. Ceritinib therapy was continued, and the patient underwent radiotherapy with VMAT technique and a total dose of 54 Gy $(25 \times 2$ Gy, boost $2 \times 2$ Gy). FDG-PET/CT after completion of radiotherapy showed a metabolically complete remission and very good morphological response of the metastatic LN (Fig. 1). ctDNA testing after radiotherapy did no longer show the ALK-resistant mutation. The absence of any mutations was confirmed 6 months later. No radiologic evidence of disease progression was detected at a follow-up of nearly 24 months, and ceritinib therapy is ongoing.

\section{Discussion}

Resistance mechanisms to ALK inhibitors include on-target alterations (e.g., ALKresistant mutations) or off-target mechanism [3]. The most common ALK-resistant mutations in patients progressing on ceritinib are ALK G1202R and F1174 C/L [4]. The value of invasive diagnostic approaches to identify resistance mechanism upon progression is currently unclear. Patients may derive benefit as the identification of the resistance mechanism enables tailoring of the next treatment. However, biopsy is not always feasible. Furthermore, a single biopsy may not adequately reflect the heterogenous character of the resistant tumor clones. Next-generation sequencing of ctDNA may be an alternative procedure, but there are concerns about its low sensitivity. In a recent trial, ctDNA identified resistance mechanism to ALK inhibition in $77 \%$ of all patients, $52 \%$ with ALK kinase domain mutations, and G1202R being the most common [5].

Upon progression on ceritinib, treatment options generally consist of changing the ALK inhibitor (e.g., lorlatinib) or switching to chemotherapy. In our case, the prerequisites for an oligoprogressive treatment concept were present: single progressive site accessible to localized treatment and excellent disease control elsewhere with ceritinib. Interestingly, we observed the absence of an G1202R mutation after radiotherapy in two independent ctDNA testings, suggesting a possible eradication of the resistant subclone. A negative PET scan provides additional evidence for this assumption.

To our knowledge, this is the first description of a successful combined treatment approach in an oligometastatic progression of ALK-rearranged non-small cell lung cancer resulting in both disease control and possible eradication of the resistant subclone.

\section{Acknowledgment}

The authors would like to thank the patient for allowing us to report her clinical information and data.

\section{Statement of Ethics}

The patient gave written consent for the publication of her case including publication of images. 


\section{Disclosure Statement}

D.K., U.R.M., B.K. and S.S. have no conflicts of interest to declare. M.P. reports personal fees from Abbvie, Astra Zeneca, BMS, Boehringer Ingelheim, Esei, MSD, Novartis, Pfizer, Roche, Takeda, Merck, Vifor, and Janssen.

\section{Funding Sources}

There was no financial support/funding for this article.

\section{Author Contributions}

M.P. made substantial contributions toward the concept and idea of this case report. D.K. has written the manuscript. B.K. has created the figure. M.P., U.R.M., B.K., and S.S. were involved in drafting the manuscript. All authors have approved the final version for publication.

\section{References}

1 Chan OSH, Lee VHF, Mok TSK, Mo F, Chang ATY, Yeung RMW. The role of radiotherapy in epidermal growth factor receptor mutation-positive patients with oligoprogression: a matched-cohort analysis. Clin Oncol (R Coll Radiol). 2017;29(9):568-75.

2 Weickhardt AJ, Scheier B, Burke JM, Gan G, Lu X, Bunn PA, Jr., et al. Local ablative therapy of oligoprogressive disease prolongs disease control by tyrosine kinase inhibitors in oncogene-addicted non-small-cell lung cancer. J Thorac Oncol. 2012;7(12):1807-14.

3 Rotow J, Bivona TG. Understanding and targeting resistance mechanisms in NSCLC. Nat Rev Cancer. 2017; 17(11):637-58.

4 Gainor JF, Dardaei L, Yoda S, Friboulet L, Leshchiner I, Katayama R, et al. Molecular mechanisms of resistance to first- and second-generation ALK inhibitors in ALK-rearranged lung cancer. Cancer Discov. 2016;6(10): 1118-33.

5 McCoach CE, Blakely CM, Banks KC, Levy B, Chue BM, Raymond VM, et al. Clinical utility of cell-free DNA for the detection of ALK fusions and genomic mechanisms of ALK inhibitor resistance in non-small cell lung cancer. Clin Cancer Res. 2018;24(12):2758-70. 